\title{
Morphological and functional carotid vessel wall properties in relation to cerebral white matter lesions in myocardial infarction patients
}

\author{
E.S.J. Kröner · J. van der Grond · J.J.M. Westenberg • \\ E.E. van der Wall · H.-M.J. Siebelink · H.J. Lamb
}

Published online: 12 May 2015

(C) The Author(s) 2015. This article is published with open access at Springerlink.com

\begin{abstract}
Objective Atherosclerotic large vessel disease is potentially involved in the pathogenesis of cerebral small vessel disease related to occurrence of white matter lesions (WMLs) in the brain. We aimed to assess morphological and functional carotid vessel wall properties in relation to WML using magnetic resonance imaging (MRI) in myocardial infarction (MI) patients.

Materials and methods A total of $20 \mathrm{MI}$ patients $(90 \%$ male, $61 \pm 11$ years) underwent carotid artery and brain MRI. Carotid vessel wall thickness (VWT) was assessed, by detecting lumen and outer wall contours. Carotid pulse wave velocity (PWV), a measure of elasticity, was determined using the transit-time method. Patients were divided according to the median VWT into two groups. Brain MRI allowed for the WML score.

Results Mean VWT was $1.41 \pm 0.29 \mathrm{~mm}$ and mean carotid $\mathrm{PWV}$ was $7.0 \pm 2.2 \mathrm{~m} / \mathrm{s}$. A significant correlation (Pearson $\mathrm{r}=0.45, p=0.046$ ) between VWT and PWV was observed. Furthermore, in the group of high VWT, the median WML score was higher as compared with the group with lower VWT (4.0 vs 3.0, $p=0.035$ ).
\end{abstract}

E.S.J. Kröner $(\bowtie)$ · E.E. van der Wall · H.-M.J. Siebelink Department of Cardiology, Leiden University Medical Center, Albinusdreef 2,

2333 ZA Leiden, The Netherlands

e-mail: e.s.j.kroner@lumc.nl

E.E. van der Wall · E.S.J. Kröner

The Interuniversity Cardiology Institute of the Netherlands, Utrecht, The Netherlands

J. van der Grond · J.J.M. Westenberg · H.J. Lamb Department of Radiology, Leiden University Medical Center, Leiden, The Netherlands
Conclusions Carotid artery morphological and functional alterations are correlated in MI patients. Patients with high VWT showed a higher amount of periventricular WMLs. These findings support the hypothesis that atherosclerotic large vessel disease is potentially involved in the pathogenesis of cerebral small vessel disease.

\section{Introduction}

Vascular risk exposure and age exert systemic effects on both the morphology and function of the arterial vessel wall by degeneration of the aortic media and breakdown of elastic fibres [1]. Accordingly, in patients with established atherosclerotic disease, e.g. patients with a previous myocardial infarction (MI), accelerated morphological changes are considered to be associated with increased arterial stiffness. Moreover, atherosclerotic large vessel disease is potentially involved in the pathogenesis of cerebral white matter lesions (WMLs) that are generally regarded as manifestations of cerebral small vessel disease [2-4]. Indeed, it is hypothesised that pathologically increased arterial stiffness results in deficient absorption of the pulse wave travelling through the vascular system. A deficient absorption of the systolic pulse wave may result in transmission of high pulsatile flow from the aorta towards the carotid artery and the brain, potentially initiating carotid vessel wall remodelling and functional changes which may lead to the development of cerebral small vessel disease and subsequent WMLs. Accordingly, the association between systemic large vessel disease, cerebral small vessel disease and WMLs in MI patients is of interest.

Noninvasive evaluation of morphological and functional vessel wall properties and cerebral WMLs is feasible using a comprehensive magnetic resonance imaging (MRI) 
approach [5-7]. Still, very little is known about the direct association between carotid vessel wall thickness (VWT), vascular stiffness and white matter brain lesions in MI patients. Therefore, the purpose of this study was to assess the association between morphological and functional carotid vessel wall properties and cerebral periventricular WML in MI patients using MRI.

\section{Materials and methods}

Population and study protocol

A total of 20 MI patients ( $90 \%$ male, $61 \pm 11$ years) who previously suffered from an MI were included. Approval from the local medical ethics committee was obtained and all patients gave written informed consent. Patients underwent 3 T MRI examinations (Philips Achieva Philips Medical Systems, Best, the Netherlands) between October 2011 and November 2012. Carotid artery VWT, pulse wave velocity (PWV) in the carotid artery and cerebral WMLs were assessed using MRI techniques [7-9] (Fig. 1).
MRI acquisition

\section{Carotid vessel wall thickness}

Carotid VWT was determined using a two-dimensional (2D) T1-weighted segmented gradient echo sequence using a standard Philips SENSE-flex-M surface coil with two flexible elements of $14 \times 17 \mathrm{~cm}$ as previously described [5]. Scan parameters: 2D black blood (BB) T1-weighted fast gradient echo sequence, field of view (FOV) $140 \times 140 \mathrm{~mm}^{2}$, $2.0 \mathrm{~mm}$ slice thickness, flip angle (FA) $45^{\circ}$, repetition time (TR) $12.4 \mathrm{~ms}$, echo time (TE) $3.5 \mathrm{~ms}$, acquired resolution $0.46 \times 0.46 \times 2.0 \mathrm{~mm}^{3}$, number of signal averages (NSA) 2 . Acquisitions were gated at end-diastole using vector ECG.

\section{Carotid pulse wave velocity}

Carotid PWV was assessed by two consecutive velocityencoded (VE) MRI acquisitions using a 16-element neurovascular head-neck coil, as previously described [8]. The first acquisition (proximal) was planned perpendicular to the left carotid artery at the level of the origin of the com-

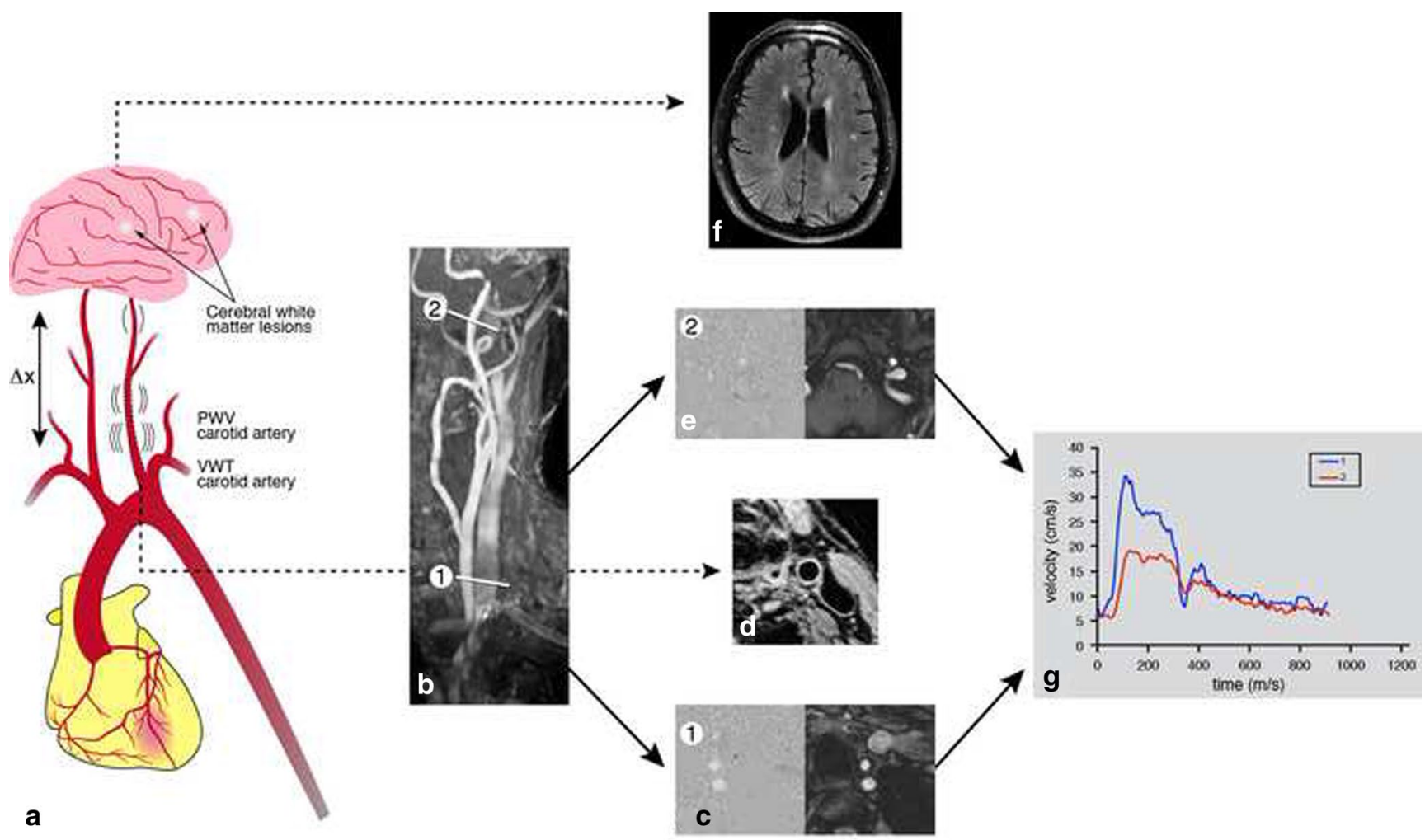

Fig. 1 A schematic representation of the study protocol (a). Carotid vessel wall properties (vessel wall thickness (VWT) and pulse wave velocity $(\mathrm{PWV})$ ) and cerebral periventricular white matter lesions (WML) were assessed in MI patients. PWV was assessed at two locations, proximally at the left common carotid artery just above the aortic arch (1) and distally just below the petrous portion of the left internal carotid artery (2), which were planned on the rotational maximum- intensity projection of a 3D time-of-flight acquisition of the carotid arteries (b). The corresponding velocity-encoded images are represented in $\mathbf{c}$, $\mathbf{e}$ for the proximal and distal acquisition respectively. From the propagation of the velocity waveforms (g), PWV is determined. VWT was assessed at the left common carotid artery (4-mm proximal to the carotid bifurcation) (d). Cerebral WML were determined using spinecho T2-weighted and a fluid-attenuated inversion recovery (f). 
mon carotid artery and the second (distal), at the level of the internal carotid artery, below the petrous segment (Fig. 1b, $\mathrm{c}, \mathrm{e})$.

Scan parameters were: FOV $200 \times 200 \mathrm{~mm}^{2}$, slice thickness $5 \mathrm{~mm}$, FA $10^{\circ}$, TR $6.2 \mathrm{~ms}$, TE $3.4 \mathrm{~ms}$, acquisition resolution $1.52 \times 1.50 \times 5.0 \mathrm{~mm}^{3}$, NSA 1 , velocity sensitivity $\left(\mathrm{V}_{\text {enc }}\right)$ for the proximal acquisition $150 \mathrm{~cm} / \mathrm{s}$ and distal acquisition $120 \mathrm{~cm} / \mathrm{s}$, both in through-plane direction. The true temporal resolution (TRes, defined as $2 \times \mathrm{TR}$, amounted to $12.4 \mathrm{~ms}$ per heart phase). Retrospective gating using vector ECG triggering was used.

\section{Cerebral white matter lesions}

Cerebral WMLs were determined using spin-echo T2-weighted and fluid-attenuated inversion recovery (FLAIR) sequences as previously described [10]. Scan parameters for T2-weighted imaging: FOV $224 \times 180 \mathrm{~mm}^{2}$, matrix size $448 \times 320,40$ transverse slices without gap, $3.6 \mathrm{~mm}$ slice thickness, FA $90^{\circ}$, TR $4200 \mathrm{~ms}$, TE $80 \mathrm{~ms}$. Scan parameters for FLAIR sequence: FOV $220 \times 176 \mathrm{~mm}^{2}$, matrix size: $320 \times 240,25$ transverse slices without gap, $5 \mathrm{~mm}$ slice thickness, FA $90^{\circ}$, TR $11000 \mathrm{~ms}$, TE $125 \mathrm{~ms}$ [10].

Image analysis

\section{Carotid vessel wall thickness}

Contour segmentation was performed on a slice of the left common carotid artery (4-mm proximal to the carotid bifurcation) using Vessel MASS software (Leiden University Medical Centre, Leiden, the Netherlands) as previously described [11] (Fig. 1d). Mean and maximal carotid vessel wall thickness $(\mathrm{mm})$ were evaluated. Contour segmentation was performed by a researcher with 3 years of experience in cardiac MRI.

\section{Pulse wave velocity}

Carotid PWV was obtained from the VE MRI data [7, 8]. The carotid artery path length $(\Delta \mathrm{x})$ between subsequent proximal and distal carotid artery sampling sites was manually determined. Wave propagation was evaluated from maximal velocity time curves that were obtained by using FLOW software (Leiden University Medical Centre, Leiden, the Netherlands) with automated contour detection for image segmentation. The foot-to-foot definition was used for $\Delta t$ (i.e. the transit time) assessment; with automated detection of the foot of the systolic velocity wave front (i.e. the wave arrival time). Accordingly, carotid PWV was calculated as $\Delta \mathrm{x} / \Delta \mathrm{t}(\mathrm{m} / \mathrm{s})($ Fig. 1g).

\section{White matter lesions}

White matter hyperintensities/lesions were defined as areas within the cerebral white matter, with increased signal on both T2-weighted images and FLAIR images without mass effect [10] (Fig. 1f). WMLs were rated according to a slightly modified version of the semi-quantitative rating scale of Scheltens et al. [12], as previously described [10]. Periventricular and subcortical WMLs were rated separately. Periventricular WMLs for three separate regions (anterior, lateral, posterior) were scored as: no white matter lesions (0); normal amount of white matter lesions (1); abnormal amount (2); very abnormal amount (3) [12]. Next, a total periventricular WML score was calculated as the sum of the three individual scores.

Subcortical WMLs were scored as: no white matter lesions (0); 1-3 small lesions (1); > 3 small lesions (2); very abnormal, confluent lesions (3) [12].

WML were scored by a researcher ( JvdG) with 15 years of experience in neuroradiology.

\section{Statistical analysis}

Statistical analysis was performed using SPSS for Windows (version 18.0; SPSS, Chicago, Illinois, USA). Data are expressed as mean \pm standard deviations (SD) unless stated otherwise. The relation between and morphological measurements (VWT in the carotid artery) and functional properties (i.e. PWV of the carotid artery) was assessed. Next, the association between vessel wall parameters and WMLs was investigated. The association between mean and maximal carotid VWT and carotid PWV was assessed using linear regression. Multivariate linear regression analysis with PWV as an independent variable and VWT and age as dependent variables was performed to assess the influence of age on the association between VWT and PWV. Next, patients were divided into groups, according to the median of VWT. Carotid PWV as well as the total amount of periventricular and subcortical WMLs was compared (using the Mann-Whitney test) between the group with high VWT $(>$ median VWT) versus the group with low VWT $(\leq$ median VWT).

\section{Results}

Patient characteristics and results are presented in Table 1. In all, 20 MI patients (18 male, 2 female, mean age $61 \pm 11$ years) were included. All patients were prescribed medication for secondary prevention of MI (i.e. anticoagulants and or platelet inhibitors, beta-blockers, angiotensin-converting enzyme inhibitors or angiotensin II inhibitors and statins) according to clinical guidelines [13]. 
Table 1 Characteristics and results of study population $(n=20)$

Age at magnetic resonance imaging (MRI) (years) $61 \pm 11$

Age range (min.-max.)

(37-82)

Days between MI and vessel wall MRI scan (days) $\quad 299 \pm 144$

Culprit vessel at MI

Left anterior descending artery $\quad 5(25 \%)$

Right coronary artery

$13(65 \%)$

Circumflex artery

Peak troponin T values $(\mu \mathrm{g} / \mathrm{l})$

$2(10 \%)$

$3.8 \pm 2.4$

$18(90 \%)$

Male gender, $\mathrm{n}(\%)$

$26 \pm 3$

BMI $\left(\mathrm{kg} / \mathrm{m}^{2}\right)$

$125 \pm 23$

Systolic

$76 \pm 12$

Diastolic

$74 \pm 16$

Heart rate (beats per minute)

$10(50 \%)$

$2(10 \%)$

$8(40 \%)$

Smokers, n (\%)

$5.09 \pm 1.17$

Total cholesterol ( $\mathrm{mmol} / \mathrm{l})$

Data are represented as mean \pm standard deviation

$M I$ myocardial infarction, $B M I$ body mass index

Table 2 Magnetic resonance imaging carotid vessel wall $(n=20)$

\begin{tabular}{ll}
\hline & $\begin{array}{l}\text { Myocardial } \\
\text { infarction } \\
\text { patients }(n=20)\end{array}$ \\
\hline Trajectory carotid artery, $\mathrm{mm}$ & $147 \pm 20$ \\
PWV carotid artery, $\mathrm{m} / \mathrm{s}$ & $7.0 \pm 2.2$ \\
Mean vessel wall thickness carotid artery, $\mathrm{mm}$ & $1.41 \pm 0.3$ \\
Maximal vessel wall thickness carotid artery, $\mathrm{mm}$ & $1.73 \pm 0.4$ \\
\hline Data are represented as mean \pm standard deviation & \\
$P W V$ pulse wave velocity &
\end{tabular}

Vessel wall morphology and function

MRI measurements are presented in Table 2. Mean carotid vessel wall thickness was $1.41 \pm 0.29 \mathrm{~mm}$ and mean carotid PWV $7.0 \pm 2.2 \mathrm{~m} / \mathrm{s}$.

Interestingly, PWV of the carotid artery was significantly correlated with VWT of the carotid artery (Pearson $r=0.45$, $p=0.046)$. The associations between PWV of the carotid artery and mean and maximal VWT are presented in Fig. $2 \mathrm{a}$ and $\mathrm{b}$. Also, the influence of age on the association between PWV and VWT is shown. Age sharpened the association between vessel wall thickness and pulse wave velocity. However, age was not a statistically significant predictor in the multiple regression model.

White matter lesions

In 15 out of 20 patients, brain MRI was also acquired; 5 (33\%) patients showed an abnormal amount of periventricular WMLs $(n=5)$, according to the modified Scheltens score; $12(80 \%)$ patients were diagnosed with the presence of subcortical WMLs. Detailed scores for anatomical subdivision are provided in Table 3 .

Association vessel wall thickness, pulse wave velocity and white matter lesions

In patients with high VWT (>median VWT), a borderline significantly higher carotid PWV $(7.1 \mathrm{~m} / \mathrm{s}$ versus $5.98 \mathrm{~m} / \mathrm{s}$, $p=0.07$ ) as compared with the patients with low vessel wall thickness was observed (Fig. 2c). Furthermore, the total periventricular WMLs score was significantly higher in the patients with high vessel wall thickness as compared with patients with low vessel wall thickness (4.0 vs 3.0, $p=0.035$ ) (Fig. 2d). In patients with high VWT, the total subcortical WMLs score was similar as compared with patients with low VWT (2.0 vs $1.0, p=0.3)$.

\section{Discussion}

The present study evaluated the association between carotid morphological and functional imaging parameters and cerebral WML in patients after MI. The main findings of our study are: (i) carotid VWT and carotid PWV are statistically significantly correlated in MI patients, and (ii) the total periventricular WMLs score was higher in the MI patients with high carotid vessel wall thickness versus the patients with low carotid VWT.

To the best of our knowledge, our study is the first to report an evaluation of both carotid VWT and PWV and cerebral WMLs in MI patients using a comprehensive MRI evaluation.

The assessment of VWT and PWV by MRI correlates to presence of WMLs, which is of clinical interest. Previous studies used echo (Doppler) for the assessment of VWT and arterial stiffness [14-16]. This imaging technique is restricted by the choice of imaging plane (i.e. only sampling in the common carotid artery is possible). In contrast, MRI allows direct sampling of VWT and PWV, without restrictions regarding the choice of imaging plane, thereby allowing sampling along the carotid arterial trajectory from the common carotid artery into the internal carotid artery. A recent MRI study by Corti et al. [17] showed a decrement in carotid vessel wall area and maximal carotid artery thickness, but not minimal carotid artery thickness in hypercholesterolaemic patients after statin use. We aimed to explore not only the morphological vessel wall changes but also the association between morphological and functional properties of the vessel wall. 
Fig. 2 Association between carotid artery pulse wave velocity and mean and maximal carotid vessel wall thickness in myocardial infarction patients (a, b). Comparison of carotid pulse wave velocity and white matter lesions in patients with low versus high carotid vessel wall thickness $(\mathbf{c}, \mathbf{d})$
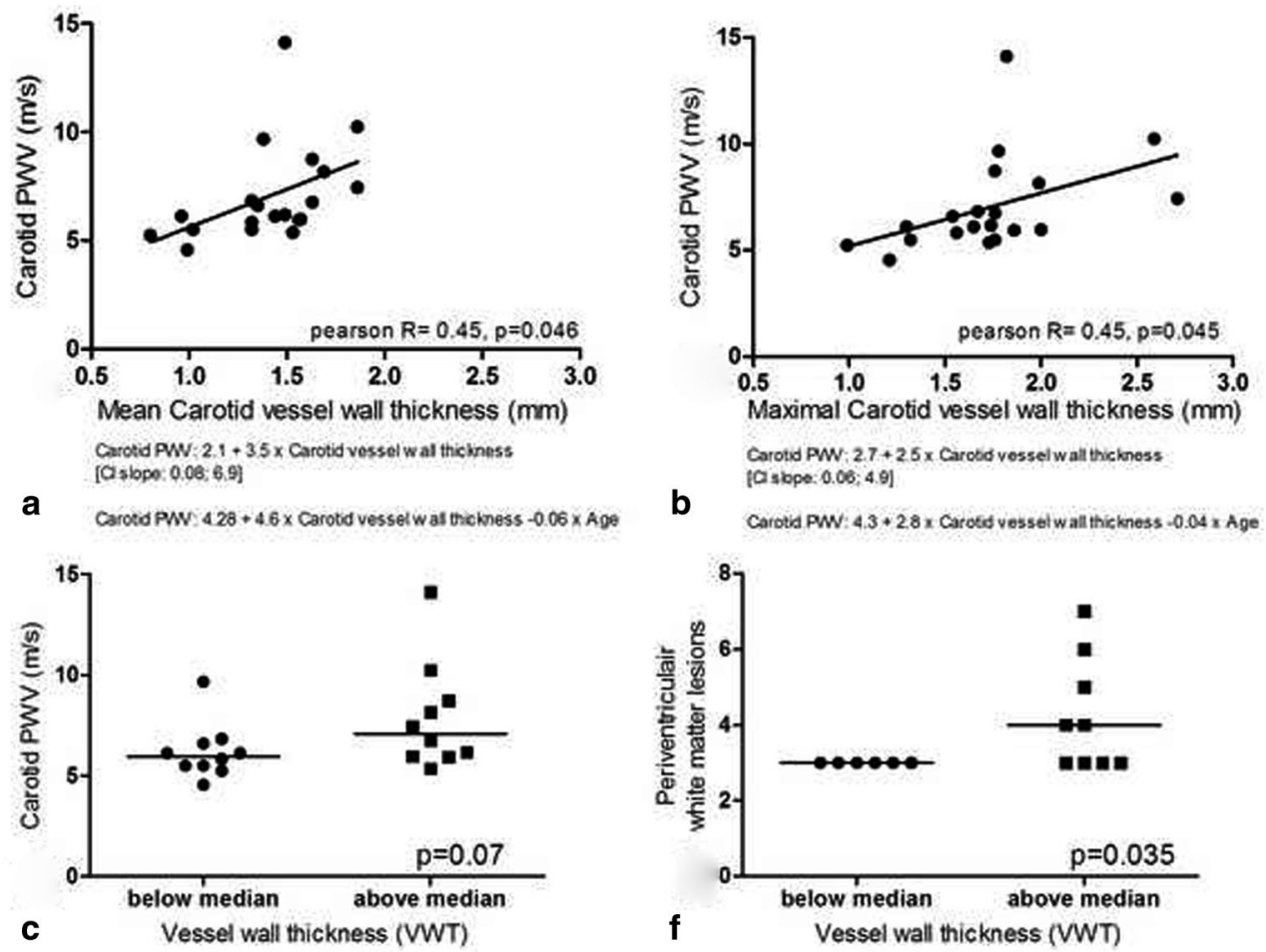

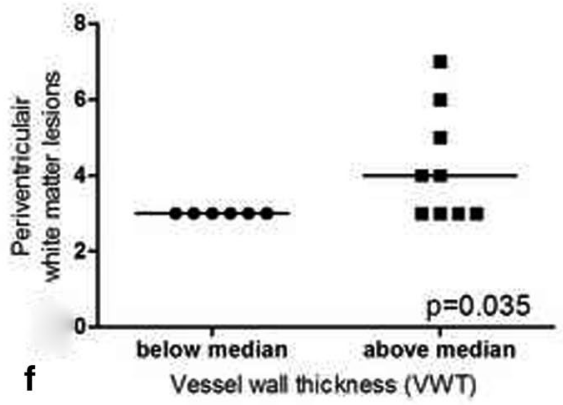

Table 3 MRI white matter lesions $(n=15)$

\begin{tabular}{lllll}
\hline & \multicolumn{2}{l}{ Periventricular lesion score } & \multicolumn{2}{l}{$\begin{array}{l}\text { Subcortical } \\
\text { lesion score }\end{array}$} \\
\cline { 2 - 4 } Anterior & Lateral & Posterior & $3(20)$ \\
Score 0 & $0(0)$ & $0(0)$ & $0(0)$ & $3(33)$ \\
Score 1 & $11(73)$ & $11(73)$ & $14(93)$ & $5(40)$ \\
Score 2 & $3(20)$ & $3(20)$ & $1(7)$ & $6(7)$ \\
Score 3 & $1(7)$ & $1(7)$ & $0(0)$ & $1(7)$ \\
\hline
\end{tabular}

Data are presented as number (percentage)

Vessel wall morphology and function

Our study showed a significant association between mean and maximal VWT and PWV in the carotid artery in patients after MI. Our findings are consistent with a previous population-based cohort study by Van Popele et al. [15], who found increased common carotid stiffness assessed by ultrasound, in the highest quartile of intima-media thickness of the common carotid artery. Interestingly, our results indicate that morphology and functional parameters remain clearly associated in patients with established atherosclerotic disease. We also observed that age influenced the association between VWT and PWV. This finding is in line with previous studies describing increased carotid artery stiffening with age $[2,14]$. Of note, in the present study, age was not a statistically significant predictor in the multiple regression model. This could potentially be explained by the relatively small study group. Accordingly, future studies remain needed to further assess the influence of age on the association between vessel wall morphology and function in patients with established atherosclerotic disease.

White matter lesions

The prevalence of WMLs observed in the present study is in line with previous studies $[10,18]$. A high prevalence of WMLs in MI patients is indeed to be expected, since risk factors associated with an MI are also risk factors for cerebral small vessel disease [19].

Increased vessel wall thickness and white matter lesions

Our study revealed that in MI patients with high VWT, the amount of periventricular WMLs was significantly higher as compared with subjects without increased VWT. Our findings are in line with a previous study in a population-based cohort ( $n=640$, age: $59-71$ years), describing the association between carotid atherosclerosis assessed by ultrasonography and WMLs [20]. Moreover, Kwee et al. [21] showed that in transient ischaemic attack (TIA)/stroke patients with carotid artery stenosis, carotid plaque burden and WML severity were associated. Our results indicate that carotid VWT and WMLs are not only associated in the elderly general population and TIA/stroke patients but also in patients with a previous MI.

In contrast to the result for periventricular WMLs, no relation between carotid vessel wall thickness and subcortical WMLs was observed. This difference is in line with a 
previous study [22] and may be explained to be the result of hypoperfusion that can be caused by large vessel disease. Due to differences in blood supply, the periventricular white matter is more vulnerable to a decrease in cerebral blood flow [10, 22].

The current study showed associations between morphological and functional carotid vessel wall properties and cerebral white matter lesions in MI patients. The presence of WMLs in MI patients may have relevant clinical implications, since WMLs have been suggested to increase the risk of stroke and cognitive decline [18]. However, it is not likely that MR imaging of the brain will be a routine investigation in MI patients. But, for identification of MI patients at risk, carotid vessel wall parameters might become beneficial in the future.

\section{Limitations}

Our study has some limitations. First, it involves a crosssectional design in a relatively small group of patients $(n=20)$, of which 15 underwent brain MRI.

Follow-up studies are needed to further elucidate pathophysiological mechanisms between large vessel atherosclerosis and small vessel disease in patients with established atherosclerotic disease.

Furthermore, the comprehensive MRI evaluation of the present study is time-consuming and relatively expensive in comparison with echocardiography. But for serial assessment of both vessel wall parameters and WML, MRI allows a noninvasive, reproducible evaluation without restriction regarding imaging plane.

\section{Conclusion}

Morphological and functional alterations in the carotid artery are significantly correlated in MI patients. Patients with high carotid VWT showed a higher amount of periventricular WMLs. These findings support the hypothesis that atherosclerotic large vessel disease is potentially involved in the pathogenesis of cerebral small vessel disease.

Acknowledgements We would like to thank Mr. Gerrit Kracht for the graphic design.

Funding ICIN- Netherlands Heart Institute.

\section{Conflict of interest None.}

Open Access This article is distributed under the terms of the Creative Commons Attribution License which permits any use, distribution, and reproduction in any medium, provided the original author(s) and the source are credited.

\section{References}

1. Lakatta EG, Levy D. Arterial and cardiac aging: major shareholders in cardiovascular disease enterprises: part I: aging arteries: a "set up" for vascular disease. Circulation. 2003;107:139-46.

2. Mitchell GF. Effects of central arterial aging on the structure and function of the peripheral vasculature: implications for end-organ damage. J Appl Physiol. 2008;105:1652-60.

3. Mitchell GF, Buchem MA, Sigurdsson S, et al. Arterial stiffness, pressure and flow pulsatility and brain structure and function: the age, gene/environment susceptibility-Reykjavik study. Brain. 2011;134:3398-407.

4. Ritz K, Buchem MA van, Daemen MJ. The heart-brain connection: mechanistic insights and models. Neth Heart J. 2013;21:55-7.

5. Alizadeh DR, Doornbos J, Tamsma JT, et al. Assessment of the carotid artery by MRI at 3T: a study on reproducibility. J Magn Reson Imaging. 2007;25:1035-43.

6. Wall EE van der. Crown years for non-invasive cardiovascular imaging (Part III): 30 years cardiovascular magnetic resonance. Neth Heart J. 2013;21:263-5.

7. Westenberg JJ, Roos A de, Grotenhuis HB, et al. Improved aortic pulse wave velocity assessment from multislice two-directional in-plane velocity-encoded magnetic resonance imaging. J Magn Reson Imaging. 2010;32:1086-94.

8. Kroner ES, Lamb HJ, Siebelink HM, et al. Pulse wave velocity and flow in the carotid artery versus the aortic arch: effects of aging. J Magn Reson Imaging. 2014;40:287-93.

9. Kroner ES, Lamb HJ, Siebelink HM, et al. Coupling of vessel wall morphology and function in the aorta and the carotid artery: an evaluation with MRI. Int J Cardiovasc Imaging, 2014;30:91-8.

10. Altmann-Schneider I, Grond J van der, Slagboom PE, et al. Lower susceptibility to cerebral small vessel disease in human familial longevity: the Leiden Longevity Study. Stroke. 2013;44:9-14.

11. Kroner ES, Schinkel LD van, Versluis MJ, et al. Ultrahigh-field 7-T magnetic resonance carotid vessel wall imaging: initial experience in comparison with 3-T field strength. Invest Radiol. 2012;47:697-704.

12. Scheltens $P$, Barkhof F, Leys D, et al. A semiquantative rating scale for the assessment of signal hyperintensities on magnetic resonance imaging. J Neurol Sci. 1993;114:7-12.

13. Liem SS, Hoeven BL van der, Oemrawsingh PV, et al. MISSION!: optimization of acute and chronic care for patients with acute myocardial infarction. Am Heart J. 2007;153:14.e1-11.

14. Paini A, Boutouyrie P, Calvet D, Tropeano AI, Laloux B, Laurent S. Carotid and aortic stiffness: determinants of discrepancies. Hypertension. 2006;47:371-6.

15. Popele NM van, Grobbee DE, Bots ML, et al. Association between arterial stiffness and atherosclerosis: the Rotterdam Study. Stroke. 2001;32:454-60.

16. Erqou S, Kip KE, Mulukutla SR, Aiyer AN, Reis SE. Racial differences in the burden of coronary artery calcium and carotid intima media thickness between Blacks and Whites. Neth Heart J. 2015;23:44-51.

17. Corti R, Osende JI, Fayad ZA, et al. In vivo noninvasive detection and age definition of arterial thrombus by MRI. J Am Coll Cardiol. 2002;39:1366-73.

18. Gerdes VE, Kwa VI, Cate H ten, Brandjes DP, Buller HR, Stam J. Cerebral white matter lesions predict both ischemic strokes and myocardial infarctions in patients with established atherosclerotic disease. Atherosclerosis. 2006;186:166-72.

19. Remmelink M, Sjauw KD, Yong ZY, et al. Coronary microcirculatory dysfunction is associated with left ventricular dysfunction during follow-up after STEMI. Neth Heart J. 2013;21:238-44. 
20. Pico F, Dufouil C, Levy C, et al. Longitudinal study of carotid atherosclerosis and white matter hyperintensities: the EVA-MRI cohort. Cerebrovasc Dis. 2002;14:109-15.

21. Kwee RM, Hofman PA, Gronenschild EH, et al. Association between carotid plaque characteristics and cerebral white matter lesions: one-year follow-up study by MRI. PLoS One. 2011;6:e17070.
22. Leeuw FE de, Groot JC de, Bots ML, et al. Carotid atherosclerosis and cerebral white matter lesions in a population based magnetic resonance imaging study. J Neurol. 2000;247:291-6.

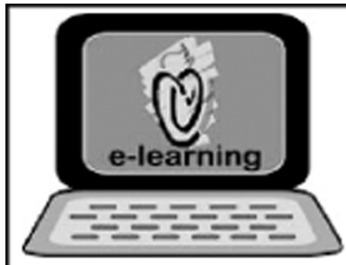

\section{CVOI E-learning formula!}

This is the CVOI e-learning article. The author has prepared 10 questions which are available through the website of the Cardiovascular Educational Institute (CVOI). Please follow the instructions below.

After finishing the questions you will be asked to fill in your name, hospital and e-mail address; then press the button 'verzenden'.

When 6 out of the 10 questions are answered correctly, you acquire 1 accreditation point granted by the Quality Committee of the Netherlands Society of Cardiology (NVVC). The acquired point will be credited to your personal file in the GAIA system. You will also receive an e-mail with all the correct answers.

Over a period of one year 10 e-learning articles will appear in 10 subsequent NHJ editions. In each edition the e-learning article will be recognisable by a special icon. On an annual basis you can collect 10 accreditation points. The accreditation points are credited in the GAIA system by the CVOI.

If you need additional information, please contact the CVOI by e-mail: cvoi@cvoi.org or by phone: 030-2345001.

E.E. van der Wall

Chief editor NHJ
K.B. Schick

Coordinator CVOI 